\title{
Sirtuins: from metabolic regulation to brain aging
}

\author{
Wenzhen Duan ${ }^{1,2,3 *}$ \\ ' Division of Neurobiology, Department of Psychiatry and Behavioral Sciences, Johns Hopkins University School of Medicine, Baltimore, MD, USA \\ ${ }^{2}$ Department of Neuroscience, Johns Hopkins University School of Medicine, Baltimore, MD, USA \\ ${ }^{3}$ Program in Cellular and Molecular Medicine, Johns Hopkins University School of Medicine, Baltimore, MD, USA
}

\section{Edited by:}

Gizem Donmez, Tufts University School of Medicine, USA

\section{Reviewed by:}

Diego Ruano, University of Seville, Spain

Douglas Watt, Quincy Medical Center, USA

Flint Beal, Weill Medical College of Cornell University, USA

\section{*Correspondence:}

Wenzhen Duan, Division of Neurobiology, Department of Psychiatry and Behavioral Sciences, Johns Hopkins University School of Medicine, CMSC 8-121, 600 North Wolfe Street, Baltimore, MD 21287, USA

e-mail:wduan2@jhmi.edu
Brain aging is characterized by progressive loss of neurophysiological functions that is often accompanied by age-associated neurodegeneration. Calorie restriction has been linked to extension of lifespan and reduction of the risk of neurodegenerative diseases in experimental model systems. Several signaling pathways have been indicated to underlie the beneficial effects of calorie restriction, among which the sirtuin family has been suggested to play a central role. In mammals, it has been established that sirtuins regulate physiological responses to metabolism and stress, two key factors that affect the process of aging. Sirtuins represent a promising new class of conserved deacetylases that play an important role in regulating metabolism and aging. This review focuses on current understanding of the relation between metabolic pathways involving sirtuins and the brain aging process, with focus on SIRT1 and SIRT3. Identification of therapeutic agents capable of modulating the expression and/or activity of sirtuins is expected to provide promising strategies for ameliorating neurodegeneration. Future investigations regarding the concerted interplay of the different sirtuins will help us understand more about the aging process, and potentially lead to the development of therapeutic approaches for the treatment of age-related neurodegenerative diseases and promotion of successful aging.

Keywords: SIRT1, sirt3, mitochondrial metabolism, brain aging, calorie restriction

\section{INTRODUCTION}

The brain, similar to other organs, undergoes a gradual decline in energy metabolism during aging (Drew and Leeuwenburgh, 2004; Navarro and Boveris, 2007; Boveris and Navarro, 2008; Swerdlow, 2011). Since neurons require large amounts of energy for the firing of action potential, neurotransmission, and other processes, the age-related decline in metabolism contributes to the cognitive declines associated with aging (Biessels and Kappelle, 2005; Boveris and Navarro, 2008). Aging is also a risk factor for age-associated diseases such as neurodegenerative disorders. These diseases may occur when neurons fail to respond adaptively to an age-related decline in basal metabolic rates and in energy-driven tasks, such as neuromuscular coordination, cognitive performance, and environmental awareness (Swerdlow, 2007). In the past decade, the function of mammalian sirtuins, evolutionarily conserved nicotinamide adenine dinucleotide (NAD)-dependent protein deacetylases/ADP-ribosyltransferases, has been investigated in greater detail, and we now have a much better molecular understanding of the multiple roles that this unique family of enzymes plays in aging and seemingly every biological process. There is little doubt that sirtuins have emerged as critical modulators of metabolic adaptive responses, and their activities have been linked to multiple diseases, from metabolic abnormalities to neurodegeneration.

Sirtuins were originally identified as one of the genes that regulate the mating types of budding yeast, Saccharomyces cerevisiae, and named silent information regulator 2 (Sir2) in lower organisms (Klar and Fogel, 1979). Following the first publication describing a role for yeast Sir2 in promoting longevity
(Kaeberlein et al., 1999), many investigations focused on elucidating whether sirtuins might play similar roles in other organisms. Sirtuins have been shown to regulate lifespan in lower organisms, including yeast, nematodes, and fruit flies (Haigis and Guarente, 2006), although their role in worm and fly lifespan has recently been debated (Burnett et al., 2011; Viswanathan and Guarente, 2011). Most of these studies have described a key role for SIRT1 in regulating the metabolic response to calorie restriction (CR; Canto and Auwerx, 2009), a dietary intervention that robustly extends life span across numerous species. However, whole body overexpression of SIRT1 in mice does not affect life span (Herranz et al., 2010). Nevertheless, SIRT1 does appear to promote healthy aging by protecting against several age-related pathologies, such as facilitating insulin sensitivity, elevating glucose production, reducing oxidative stress, potentiating activity of brain-derived neurotrophic factor (BDNF) transcriptional factor cAMP response element-binding protein (CREB; Guarente and Franklin, 2011).

Mammals have seven sirtuins (SIRT1-7) which are found in different subcellular locations, including the nucleus (SIRT1, SIRT6, and SIRT7), cytosol (SIRT2), and mitochondria (SIRT3, SIRT4, and SIRT5). Most of the studies have described a key role for SIRT1 in regulating the metabolic response to CR (Canto and Auwerx, 2009), a dietary regimen involving reduced 30-40\% calorie intake compared to normal calorie intake, that resulted in extended lifespan and reduced development of morbidity with aging (Jiang et al., 2000; Masoro, 2000; Sinclair, 2002; Koubova and Guarente, 2003). Calorie restriction is the only intervention that has consistently been shown to delay the onset, slow the progression of age-related 
disease, and extend lifespan in short-lived species, as well as in long-lived non-human primates, suggesting that similar mechanisms would be operative in humans. Whole body overexpression of Sirt1 in mice does not affect lifespan (Herranz et al., 2010). Nevertheless, SIRT1 promotes healthy aging by preventing ageassociated pathologies (Guarente and Franklin, 2011). Another strong link between mammalian sirtuins and the anti-aging effects of CR was provided by SIRT3, which mediates the prevention of age-related hearing loss (Someya et al., 2010). SIRT3 is required for the CR-mediated reduction of oxidative damage in multiple tissues via regulation of the glutathione antioxidant system (Someya et al., 2010).

In this review, we focus on the effects of SIRT1 and SIRT3 on metabolic regulation and their anti-aging activity in brain, and further discuss potential pharmacological approaches to remedy and prevent age-associated neurological disorders by targeting sirtuins.

\section{SIRT1, METABOLISM AND BRAIN AGING DISTRIBUTION OF SIRT1 IN THE BRAIN}

During mouse embryogenesis, SIRT1 is highly expressed in the brain, spinal cord, and dorsal root ganglion, with the peak expression at E4.5 (Salminen and Kaarniranta, 2012). SIRT1 is also expressed in the adult brain, with high levels in the cortex, hippocampus, cerebellum, and hypothalamus, and low levels in white matter (Singh, 2004). Among the various cell types of brain, SIRT1 is predominantly, if not exclusively, expressed in neurons (Singh, 2004; Adler et al., 2007; Salminen and Kaarniranta, 2012). The only exception is that SIRT1 is found in microglia when cocultured with neurons (Schmitz et al., 2004). At the subcellular level, SIRT1 is viewed as a nuclear protein (Chen and Greene, 2003). Yet it is reported that SIRT1 has both nuclear import and export sequences, and that SIRT1 is present in the cytosolic fraction of mouse brain, although its cytosolic function is just beginning to be elucidated (Chen et al., 2005b; Lee et al., 2008; Hardie, 2011).

\section{SIRT1 MEDIATES METABOLIC BENEFITS UNDER CR}

SIRT1 contains 747 amino acids in humans, with a predicted molecular weight of $81 \mathrm{kDa}$ and a measured one of $120 \mathrm{kDa}$. In addition to histones, SIRT1 also deacetylates a number of nonhistone substrates, including p53 (Luo et al., 2001)and peroxisome proliferator-activated receptor $\gamma(\operatorname{PPAR} \gamma)$ coactivator- $1 \alpha$ (PGC$1 \alpha$; Nemoto et al., 2005), and FOXO (Xiong et al., 2011), nuclear factor $\kappa$-light-chain-enhancer of activated B cells (NF- $\kappa$ B; Salminen et al., 2008b). SIRT1 is drawing even more attention since it is considered to be one of the determining factors in lifespan extension induced by $\mathrm{CR}$, a phenomenon observed in phylogenetically diverse organisms including yeast, worm, fruit fly, and mouse (Kaeberlein et al., 1999; Tissenbaum and Guarente, 2001; Howitz et al., 2003; Rogina and Helfand, 2004). Its beneficial roles are further supported by the findings that putative SIRT1-activating compounds, such as resveratrol, also promote longevity in several species, including yeast (Howitz et al., 2003), worm (Wood et al., 2004), and mouse (Baur et al., 2006), making it an anti-aging target.
The effects of SIRT1 on longevity rely on its enzymatic activity of deacetylation of histone and non-histone substrates. While the deacetylation of histones leads to their interaction with DNA and consequent gene silencing (Braunstein et al., 1993; Sauve et al., 2006; Dali-Youcef et al., 2007), the deacetylation of non-histone proteins has a wide range of biological effects, including metabolic adjustment, survival promotion, and autophagy (Campisi, 2005; Dali-Youcef et al., 2007; Brooks and Gu, 2009; Madeo et al., 2010). For example, SIRT1 inhibits p53 (Luo et al., 2001), reducing its pro-apoptotic effect. It also inhibits NF- $\mathrm{B}$ (Yeung et al., 2004), reducing its pro-inflammatory effects. In contrast, SIRT1 activates a transcriptional coactivator, PGC-1 $\alpha$ (Nemoto et al., 2005), leading to increased glucose levels, insulin sensitivity, and mitochondrial biogenesis. Together, these and other effects, contribute to the longevity evoked by CR (Figure 1).

These metabolic changes and the cytoprotective involvement of CR are generally considered to occur in non-neural organs, such as the liver, pancreas, muscle, and fat tissues (Brooks and $\mathrm{Gu}$, 2009; Imai and Guarente, 2010). However, recent studies suggest that the hypothalamus may also contribute to the longevity effects of SIRT1 and CR via coordination of neurobehavioral and neuroendocrine changes, including body temperature, appetite, and overall physical activity (Dietrich et al., 2010; Satoh et al., 2010). SIRT1 is abundantly expressed in several regions in the hypothalamus of mice, especially in the arcuate, paraventricular, ventroand dorsomedial nuclei; and CR increases SIRT1 levels in the hypothalamus, which increases body temperature, food intake, and physical activity (Ramadori et al., 2008; Dietrich et al., 2010; Satoh et al., 2010). SIRT1 appears to be required for the aforementioned behavioral changes, which are prevented if SIRT1 is knocked out or inhibited (Chen et al., 2005a; Satoh et al., 2010). In addition to the hypothalamus, SIRT1 is also expressed in other regions of the brain, including the cortex, striatum, and hippocampus (Ramadori et al., 2008). Shortly after this finding, a neuroprotective role of SIRT1, has been reported (Tang, 2009; Morris et al., 2011).

\section{SIRT1 AND FACTORS INVOLVED IN CR AND AGING}

SIRT1 has been found to delay aging and promote longevity by regulating the activity of key cellular proteins like p53, FOXO and Ku70 that are involved in either apoptotic processes or cellular repair mechanisms. SIRT1 may thus promote health and longevity partly by either decelerating cell death and/or by boosting repair mechanisms in the cells (Wang et al., 2010).

It has become increasingly evident that the salutary effects of the $\mathrm{CR}$, are in part due to the promotion of sirtuins (Wang et al., 2010). The expression levels of SIRT1 increase upon CR in several rodent and human tissues, including white adipose, liver, skeletal muscle, brain, and kidney. Levels of NAD have been shown to rise in liver cells under CR-like conditions, which in turn induces expression of SIRT1 (Rodgers et al., 2005). SIRT1 ends up consuming NAD+ as a result of its deacetylase activity, generating nicotinamide, an inhibitor of its own activity. NAD + is known to protect neurons (Liu et al., 2009) and thus by increasing the levels of NAD+, CR may preserveSIRT1 activity. SIRT1 also activates PGC1 $\alpha$ (Rodgers et al., 2005) which results in mitochondrial biogenesis (Liu et al., 2009). A decline in mitochondrial activity 


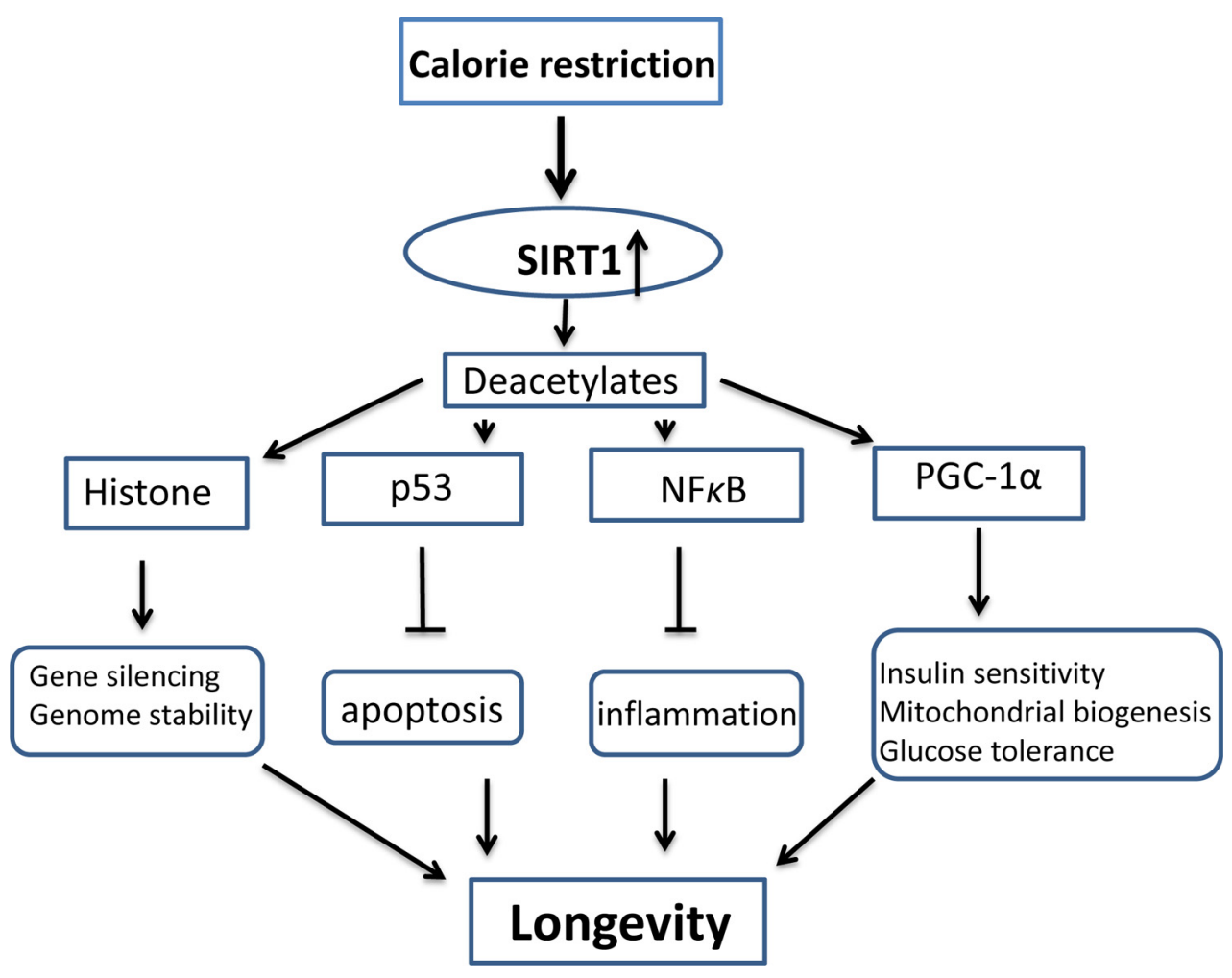

FIGURE 1 | Schematic diagram of anti-aging mechanism activated by SIRT1 and calorie restriction. Calorie restriction upregulates the activity and levels of SIRT1, SIRT1 deacetylates its substrates, including histone and non-histone molecules, and improve genome stability, prevent apoptosis and inflammation, and increases mitochondrial biogenesis, insulin sensitivity and glucose tolerance. All these effects result in longevity. is thought to be causative in many age-related diseases (Petersen et al., 2003; Singh, 2004). CR evokes improvements in mitochondrial activity similar to those of SIRT1. Therefore, it is possible that small-molecule modulators of SIRT1 may act on the same pathways as those modified by $\mathrm{CR}$, and thus have potential to mitigate age-related diseases (Lavu et al., 2008).

SIRT1 interacts with and modulates other key factors involved in mammalian aging, such as NF- $\mathrm{B}$ that controls a low-grade systemic inflammation along with human aging process-inflammaging (Salminen et al., 2008a), mammalian target of rapamycin (mTOR; Finley and Haigis, 2009), AMP-activated protein kinase (AMPK; Salminen and Kaarniranta, 2012), therefore controls the gin process. The aging process involves changes in immune regulation; NF- $\kappa \mathrm{B}$ signaling is the master regulator of the immune system. Inhibition of NF- $\kappa \mathrm{B}$ signaling in aged mice reverted the tissue characteristics and global gene expression to those of young mice (Adler et al., 2007). The function of the NFKB complex can be regulated by the acetylation of the p65 component (Schmitz et al., 2004). SIRT1 can interact with RelA/p65 protein in the NF$\kappa \mathrm{B}$ complex and specifically deacetylates lysine 310 , which has been demonstrated to potentiate the transactivation capacity of the NFкB complex (Chen and Greene, 2003). Several studies have indicated that SIRT1 is a potent inhibitor of NF- $\kappa \mathrm{B}$ transcription (Yeung et al., 2004; Chen et al., 2005b). The signaling link between SIRT1 and NF- $\kappa \mathrm{B}$ is especially interesting with respect to aging, as a consequence of the release of the SIRT1 brake, the transactivation efficiency of NF-кB factor is potentiated, which evokes immune activation and inflamm-aging.

Aging process is also regulated by autophagy. It has been identified the signaling pathways that regulate autophagic degradation and SIRT1 is a potent regulator of autophagic degradation (Lee et al., 2008), SIRT1 can interact with and deacetylates several components in the complexes of forming autophagosomes, such as Atg5, Atg7, and Atg8 proteins (Lee et al., 2008). There is a clear overlap between the signaling networks regulating both aging and autophagocytosis, which emphasizes the important role of autophagy in the regulating of aging and age-related degenerative diseases. It is evident that increase in autophagy can extend lifespan. mTOR activity is suppressed by CR, reduction in mTOR signaling is a logic candidate mechanism for the antiaging benefits of CR. Through deacetylation of a variety of proteins involved in autophagy process, SIRT1 can regulate physiological process during aging and moderated by $\mathrm{CR}$ (Haigis and Guarente, 2006).

Efficient control of energy metabolic homeostasis is a hallmark of improved healthspan and extended lifespan. The AMPK and SIRT1 signaling pathways are highly conserved energy sensor of increased levels of AMP and $\mathrm{NAD}^{+}$, respectively, AMPK signaling is involved in the regulation of energy metabolic homeostasis (Hardie, 2011). Canto and Auwerx (2009) demonstrated that the activation of AMPK stimulated the functional activity of SIRT1 by increasing the intracellular concentration of $\mathrm{NAD}^{+}$. Interestingly, 
SIRT1 was able to deacetylate LKB1 kinase which subsequently increased its activity (Lan et al., 2008). Since LKB1 is an upstream activator of AMPK, this signaling pathway stimulates the activation of AMPK. This positive feedback loop between SIRT1 and AMPK can also potentiate the function of the other AMPKactivated signaling pathways. The close relationship between AMPK and SIRT1 is evidence that energy balance effectively controls cellular responses via an integrated signaling network. AMPK can inhibit the activity of mTOR complex via two different mechanisms, either by directly phosphorylating the Raptor, a regulatory component of $\mathrm{mTORC} 1$, or by the phosphorylation of tuberous sclerosis protein 2 (TSC2), which subsequently suppresses the activity of mTOR (Jung et al., 2010; Mihaylova and Shaw, 2011). Taken together, SIRT1 interacts with other key anti-aging signaling pathways thereby contributing to longevity control.

It has been established that aging is a known risk factor for many neurodegenerative diseases including Alzheimer's disease (AD), Parkinson's disease (PD), Wallerian neurodegeneration, Huntington's disease (HD), and amyotrophic lateral sclerosis (ALS). The pathomechanisms involved in these disorders involve common biochemical pathways and processes, including protein misfolding, oligomerization, and aggregation, proteolysis, posttranslational modifications, mitochondrial dysfunction, abnormal metabolic processes, and proinflammatory and proapoptotic responses that we discuss in the next section.

\section{SIRT1 AND AGE-ASSOCIATED NEUROLOGICAL DISEASES Wallerian degeneration}

Wallerian degeneration refers to axonal death and degradation after focal injury, followed by breakdown of myelin sheath. The neuroprotective effect of SIRT1 against Wallerian degeneration was first discovered in wlds transgenic mice (Perry et al., 1990). These mice exhibited a significant delay in axonal degeneration after physical or chemical injury. The mechanistic basis for the delayed axonal damage was apparently associated with the mutant wlds chimeric protein. It has been shown that Nicotinamide mononucleotide adenylyltransferase 1 (NMNAT-1) activity plays an important role in the prevention of axonal damage, exerting its protective effects through SIRT1 activation, as the neuroprotection is blocked by the SIRT1 inhibitor sirtinol or siRNA-mediated SIRT1 silencing (Araki etal., 2004; Sasaki et al., 2009; Babetto et al., 2010). The role of SIRT1 remains controversial, however, as both SIRT1-dependent (Araki et al., 2004) and -independent mechanisms are reported (Wang et al., 2005b).

\section{Alzheimer's disease}

Alzheimer's disease is a terminal neurodegenerative disease, causing neuronal death and brain atrophy. The pathological hallmarks of $\mathrm{AD}$ are the intracellular tangles and extracellular plaques in brain. The tangles, also known as neurofibrillary tangles, are formed by accumulation of insoluble tau proteins, and the plaques are deposits of $\beta$-amyloid (A $\beta$ ) peptides, typically consisting of 40-42 amino acid residues.

The protective effect of SIRT1 against AD was initially observed in $C R$ studies, where $C R$ reduced $A \beta$ and plaque generation in the brains of transgenic AD mice (Patel et al., 2005; Wang et al., 2005a). Similarly, the reduction of $A \beta$ was also noticed in the cortex of fasted squirrel monkeys and is inversely correlated with SIRT1 levels (Qin etal., 2006a). These studies imply that SIRT1 is involved in neuroprotection against AD. Indeed, recent studies demonstrate that SIRT1 activation reduces the neuronal death and brain atrophy that are characteristic of AD (Chen et al., 2005b; Qin et al., 2006b; Kim et al., 2007; Donmez et al., 2010; Min et al., 2010). SIRT1 deficiency is associated with increased levels of phosphorylated-tau in neurons (Min et al., 2010) and the amount of neurofibrillary tangles in AD brains (Julien et al., 2009).

Moreover, recent studies show that either administration of resveratrol or overexpression of SIRT1 reduces $A \beta$ levels both in vitro and in vivo (Chen et al., 2005b; Qin et al., 2006b; Donmez et al., 2010). SIRT1 overexpression stimulates the production of $\alpha$-secretase in neurons and mice by two pathways: activating the retinoic acid receptor (RAR; Donmez et al., 2010) and inhibiting the rho-associated, coiled-coil-containing protein kinase 1 (ROCK1; Qin et al., 2006b). Increased levels of $\alpha$-secretase enhance normal process of Amyloid precursor protein (APP), leading to decreased generation of toxic $A \beta$. In addition, SIRT1 also reduces the NF-kappaB pathway in microglia and decreases A $\beta$ level (Chen et al., 2005b). Taken together, these results establish that SIRT1 protects against AD by multiple mechanisms, including degradation of tau and reducing levels of $A \beta$.

\section{Parkinson's disease}

Parkinson's disease is a common neurodegenerative disease caused by the death of dopaminergic neurons of the substantia nigra in the midbrain. The major symptoms of PD are rigidity, tremor, and bradykinesia. Our early study found that CR or use of 2-deoxyD-glucose, a glucose analog, reduces the loss of dopaminergic neurons in mice and improves motor function, implying that SIRT1 may be involved in the protection (Duan and Mattson, 1999). The levels of SIRT1 in dopaminergic neurons are sharply decreased by treatment with neurotoxins, such as rotenone, 6-hydroxydopamine, $\alpha$-synuclein, or 1-methyl-4-phenyl-1,2,3,6tetrahydropyridine (MPTP; Alvira et al., 2007; Pallas et al., 2008; Albani et al., 2009), which are agents widely used to model PD. Additionally, SIRT1 overexpression (Wareski et al., 2009) or activation by resveratrol (Okawara et al., 2007; Chao et al., 2008; Albani et al., 2009) slows neuronal death as well as neurodegeneration in PD models both in vivo and in vitro (Donmez et al., 2012), indicating a neuroprotective role of SIRT1 against PD. Not all studies showed a protective role of SIRT1, however. For example, no protection was observed in an MPTP-induced PD model in SIRT1 transgenic mice (Kakefuda etal., 2009). Nevertheless, despite the controversy, most research demonstrates a protective role of SIRT1 against PD, although the mechanisms are unclear.

\section{Huntington's disease}

Huntington's disease is an autosomal dominant hereditary disease with onset in middle-age. It is caused by a trinucleotide repeat mutation in the huntingtin gene that results in an increased number of glutamine residues in the N-terminus of the huntingtin protein which causes abnormal protein aggregation and 
ultimately neuronal death. Our previous study showed that CR could ameliorate the motor phenotype and extend survival of HD mice (Duan et al., 2003), indicating that pathways related to energy metabolism can modify disease progression in the disease. $\mathrm{CR}$ increases mitochondrial biogenesis by inducing endothelial nitric oxide synthase (eNOS), and NO can activate the SIRT1 gene (Nisoli et al., 2005; Haigis and Guarente, 2006), which is the mammalian ortholog of yeast Sir2, and a highly conserved $\mathrm{NAD}^{+}$-dependent protein deacetylase. Moreover, SIRT1 has been suggested to mediate some beneficial effects of CR (Canto and Auwerx, 2009; Wakeling et al., 2009; Shimokawa and Trindade, 2010; Chalkiadaki and Guarente, 2012).

The first report demonstrating the connection between SIRT1 and HD came from studies by Parker et al. (2005), who found that overexpression of Sir2.1 or treatment with resveratrol rescued neuronal dysfunction phenotypes induced by mutant polyglutamine in Caenorhabditis elegans. In contrast to the neuroprotective effect of Sir2.1 in C. elegans, Pallos et al. (2008) reported that $50 \%$ reduction of Sir2 extended survival and preserved neurons containing photoreceptor in flies expressing mutant Htt. Interestingly, in the fly model system, overexpression of Sir2 does not reduce the lethality or the level of neuronal degeneration caused by mutant Htt. Studies in both C. elegans and Drosophila suggest that complete loss of Sir2 is deleterious in the worm (Parker et al., 2005) and is deleterious compared with heterozygous loss in mutant Htt-challenged flies (Pallos et al., 2008). Although heterozygous loss of Sir2 is protective in flies, heterozygous loss of Sir2 in worms was not examined. Nevertheless, reduction of Sir2 neither alters the life-span of flies not expressing Htt nor siblings expressing Htt. Several aspects of the role of sirtuins in lifespan in C. elegans and Drosophila are controversial, and studies have indicated that Sir2 overexpression did not increase lifespan and that dietary restriction increased lifespan in flies independently of dSir2 (Burnett et al., 2011). Nonetheless, overexpression of Sir2 increases the longevity of normal flies and the longevity of diseased flies is slightly increased by elevated Sir2 (Pallos et al., 2008). The different results might be due to the amount of Sir2, its activation status, and different downstream targets involved. These controversial results warrant further investigation of the role of SIRT1 in mammalian systems.

Indeed, two independent studies by our group (Jiang et al., 2011) and Krainc's group (Jeong et al., 2011) demonstrated that modulating the levels of SIRT1 has therapeutic benefit in three different HD mouse models, and putative downstream targets of SIRT1 involved in improved disease outcomes are also identified. These two studies provide compelling support to the view that SIRT1 provides beneficial effects in HD mouse models, but also raise important questions. It is possible that the contradictory results on the effects of SIRT1 in models of HD might be explained by different effector pathways or mechanisms and by context-dependent effects or different levels of SIRT1 activation. SIRT1 has numerous targets, and different models of HD display different phenotypes by activating various targets and mechanisms. Therefore, it is not surprising to observe contradictory data, especially in different species and different models.

\section{AMYOTROPHIC LATERAL SCLEROSIS}

Amyotrophic lateral sclerosis is a chronic, fatal neurodegenerative disease, characterized pathologically by the death of motor neurons in the spinal cord and cortex, possibly induced by a deficiency in the enzyme superoxide dismutase 1 (SOD1; Rosen, 1993). In the animal model of ALS where a mutant form of SOD1 is expressed, SIRT1 levels are upregulated in motor neurons (Kim et al., 2007). SIRT1 overexpression protects neurons against toxicity induced by the mutant SOD1 in both cultured neurons and mouse brain (Kim et al., 2007). This protection corresponds to the increased deacetylation of p53. Resveratrol also enhances the protective effect of SIRT1 in a mouse model of ALS (Kim et al., 2007; Markert et al., 2010), but multiple doses are appear to be necessary to improve neurological function and increase the longevity of mice (Markert et al., 2010).

\section{Multiple sclerosis}

Multiple sclerosis is a myelin sheath disease with lesions typically located in the brain, spinal cord or cranial nerves, and, most commonly, in the optic nerve. The causes of multiple sclerosis are not fully identified but likely arise from an autoimmune etiology; therefore, it is traditionally treated as an inflammatory disease. Recently, however, multiple sclerosis has also been considered to be a neurodegenerative disease because of the co-existence of permanent axonal damage, neuronal loss, and neurological disability in patients with the disease (Lassmann, 2010; Shindler et al., 2010). In a mouse model of multiple sclerosis, experimental autoimmune encephalomyelitis (EAE), SIRT1 activation by SRT501 or SRT1720 maintains axonal density, prevents neuronal loss, and improves neuronal dysfunction (Shindler et al., 2007, 2010). SIRT1 inhibition with Sirtinol attenuates the neuroprotective effects of SRT501 (Shindler et al., 2010), suggesting a protective role of SIRT1 in multiple sclerosis. However, further investigations are necessary to fully delineate the role of SIRT1 in multiple sclerosis.

\section{Cerebral ischemia}

Ischemic stroke is a common neurological disease caused by the sudden reduction or cessation of blood flow to the brain, leading to infarction. The clinical management of stroke is difficult and current drugs must be administered within a limited time window after the onset of the stroke to provide clinical benefit. Promising candidates for neuroprotective strategies include preconditioning, mild hypothermia, and the use of chemical and biological compounds targeting critical molecular mediators of neuronal death and survival.

The neuroprotective effect of SIRT1 was first reported in ischemic preconditioning and the SIRT1 activating compound resveratrol reduced neuronal injury of the hippocampus in global cerebral ischemia in rats. Increased SIRT1 activity was also shown to be a common mechanism for the protective effects of preconditioning and resveratrol (Raval et al., 2006; Morris et al., 2011). Sirtinol, an inhibitor of SIRT1 activity, abolished the neuroprotection of preconditioning and resveratrol (Raval et al., 2006), indicating that SIRT1 plays a key role in mediating neuroprotection. This neuroprotective role is further supported by two recent studies (Chong and Maiese, 2008; Della-Morte et al., 
2009) showing that SIRT1 activation reduces ischemic neuronal injuries.

Another study showed that, in primary neuronal culture, pretreatment with $\mathrm{NAD}^{+}$pretreatment markedly reduces neuronal death induced by oxygen-glucose deprivation, an in vitro model of ischemia (Wang et al., 2008). SIRT1 is necessary for $\mathrm{NAD}^{+}$neuroprotection, as $\mathrm{NAD}^{+}$treatment upregulates SIRT1 expression and activity, and SIRT1 knockdown attenuates the protection mediated by $\mathrm{NAD}^{+}$(Wang et al., 2008). NAMPT overexpression reduces ischemic infarct, whereas NAMPT inhibition aggravates ischemic injuries. The protective effect of NAMPT is SIRT1-dependent, as SIRT1 knockout blocks the protection (Wang et al., 2011).

Despite the aforementioned evidence, controversy exists over the protective effect of SIRT1 against ischemia. In a study with SIRT1 transgenic mice, where human SIRT1 was overexpressed under the control of rat neuron-specific enolase promoter, no neuroprotection was observed against stroke as SIRT1 and wildtype mice demonstrated almost indistinguishable infarct volumes and neurological deficiency scores (Kakefuda et al., 2009). The discrepancy between this study and the others was probably due to the sustained high level of SIRT1, because it may consume too much or even deplete $\mathrm{NAD}^{+}$, which could exaggerate neuronal injury (Wang et al., 2008; Kakefuda et al., 2009; Liu et al., 2009). Therefore, it is possible that $\mathrm{NAD}^{+}$deficiency compromised the neuroprotective effect of SIRT1. In another study, nicotinamide, a compound that inhibits SIRT1 action, showed neuroprotection against ischemic injury, implying that SIRT1 might have a detrimental effect against stroke (Chong et al., 2005). However, this report might overlook other functions of nicotinamide, including that of precursor for $\mathrm{NAD}^{+}$synthesis. In fact, the same group later reported that SIRT1 overexpression prevents neurons from apoptosis after oxidative stress (Chong and Maiese, 2008).

\section{SIRT1 IN CLINICAL PRACTICE}

SIRT1-activating compounds have not yet been proven to be clinically useful for the treatment of neurodegenerative diseases. Preclinical studies have been performed in various neurodegenerative disease models, however. The information obtained from such studies could prove to be valuable for designing SIRT1-activating molecules that may be more likely to be useful clinically. The discovery of such molecules is becoming increasingly important, considering the limitations of genetic manipulations and the lack of unequivocal evidence of specific SIRT1 activation by prototype molecules like resveratrol (Sauve, 2009).

Several rational strategies based on the available protein structure and the catalytic pathways have been designed to develop small molecules that selectively activate sirtuins (Sauve, 2009). One strategy involves designing resveratrol-like molecules, which has not yielded successful results as the in vivo mechanism of SIRT1 activation is not fully understood. Another approach aims to increase the cellular levels of NAD + in order to activate SIRT1 function. This approach has the advantage of harnessing a natural metabolic pathway to enhance SIRT1 functions. Moreover, naturally occurring metabolites present the least risk of toxicity. The efficacies of agents that have been used to enhance $\mathrm{NAD}^{+}$ are still questionable, however, and $\mathrm{NAD}^{+}$enhancement affects a host of other physiological pathways, so that the approach is not specific to SIRT1. A third strategy currently in the proofof-principle stage designated nicotinamide derepression is based on countering the inhibitory effect of nicotinamide on sirtuins by designing molecules that are antagonistic to nicotinamide. This approach is still in its infancy and has not provided compounds with desired potency, but is an attractive strategy to develop further. Details of efforts to discover SIRT1-activating molecules have recently been comprehensively reviewed by Blum et al. (2011).

\section{FUTURE PERSPECTIVES ON SIRT1}

Over the last decade, our understanding of the biology of sirtuins has vastly increased from its original description as an $\mathrm{NAD}^{+}$dependent class III histone deacetylase that can control the lifespan of yeast. Of particular interest is the discovery that SIRT1 not only deacetylates histones, but also some well-known transcriptional regulators, thereby modulating a wide array of biological processes. An exciting aspect is that SIRT1 mediates neuroprotection against both acute and chronic neurological diseases. Importantly, SIRT1 activity is enhanced by small-molecule compounds; therefore, development of small-molecule activators could lead to novel therapies against neurological diseases. One of the broad protective mechanisms of SIRT1 is to suppress genome-wide gene transcription via histone deacetylation. Furthermore, SIRT1 selectively suppresses genes involved in fat storage, apoptosis, and inflammation. Adding to the complexity of SIRT1-mediated cell survival, SIRT1 specifically promotes the transcription of a set of genes related to cell survival, energy metabolism, and mitochondrial biogenesis. SIRT1 thus has multifaceted mechanisms with the end goal to increase cell viability.

Although extensively studied, the biological functions of SIRT1 remain only partially characterized. With respect to mechanism of action, there are several substantial unknowns. For example, it is not known how SIRT1 specifically increases transcription of beneficial genes while it simultaneously suppresses universal transcription. It would be of interest to determine whether similar mechanisms exist for genes upregulated by SIRT1-mediated activation of transcription. Another issue is the paradoxical effect of SIRT1. For example, whereas SIRT1 directly suppresses PPAR $\gamma$ transcriptional activity directly (Picard et al., 2004), it also activates PGC- $1 \alpha$ (Nemoto et al., 2005; Rodgers et al., 2005), which could increase the transcriptional activities of PPAR $\gamma$ (Puigserver et al., 1998).

Although SIRT1 has been found to be neuroprotective in numerous studies, it is clear from the diverse pathological mechanisms manifested in neurodegenerative disorders that the role of SIRT1 requires more detailed study. The availability of crystal structures and detailed mechanistic analysis are helpful in discovering SIRT1 modulators, but would be of limited value if they failed to reach clinical trials, thus emphasizing the importance of developing robust animal models for investigating molecular mechanisms involved in SIRT1 activation. Moreover, the potential negative effects of SIRT1 activation and energy depletion need further investigation in animal models. The clinical success of sirtuin activating compounds (STACs) in neurodegenerative diseases relies overwhelmingly on developing new strategies and designing molecules based on the sirtuin chemistry and molecular 
pathways activated by SIRT1. A very recent study performed by Hubbard et al. (2013) demonstrated that the specific hydrophobic motifs in SIRT1 substrates such as PGC- $1 \alpha$ and FOXO3a facilitate SIRT1 activation by STACs, implying that SIRT1 can be directly activated through an allosteric mechanism common to chemically diverse STACs. In summary, there is no doubt that SIRT1 holds promising therapeutic potential for neurodegenerative disorders.

\section{SIRT3, ENERGY METABOLISM AND AGING}

This section summarizes the studies on the role of mitochondrial SIRT3 in energy metabolism and protection against oxidative stress and age-associated dysfunction (Figure 2).

\section{SIRT3 AND MITOCHONDRIA}

Mitochondria are not only the powerhouse for ATP production but also the main sites where reactive oxygen species (ROS) are generated and the intrinsic apoptotic signaling pathway is initiated (Salminen et al., 2008b). The functions of mitochondrial proteins are altered when they are deacetylated by $\mathrm{NAD}^{+}$-dependent mitochondrial deacetylases, including SIRT3, SIRT4, and SIRT5. All mitochondrial sirtuins are present in the mitochondrial matrix (Howitz et al., 2003; Rogina and Helfand, 2004). Since mitochondria contain their own DNA, transcription factors, histone-like proteins, and protein synthesis systems, mitochondrial sirtuins deacetylate a set of targets within the mitochondria that are distinct from those of nuclear proteins (Tissenbaum and Guarente, 2001; Wood et al., 2004). Although precise mechanistic information is still lacking, evidence is emerging to suggest that mitochondrial sirtuins protect against oxidative stress (Braunstein et al., 1993; Baur et al., 2006).

Among the mitochondrial sirtuins, SIRT3 functions have been characterized in the greatest detail. Initial studies of SIRT3deficient mice indicated that loss of SIRT3, but not SIRT4 or SIRT5, led to dramatic protein hyperacetylation within mitochondria, suggesting that SIRT3 is the major mitochondrial deacetylase activity (Dali-Youcef et al., 2007). In humans, full-length SIRT3 is a $44-\mathrm{kD}$ protein with an $\mathrm{N}$-terminal mitochondrial targeting sequence that is an enzymatically inactive in vitro. It is proteolytically processed in mitochondria to a mature $28 \mathrm{kD}$ catalytically active deacetylase (Onyango et al., 2002; Schwer et al., 2002). The first mouse SIRT3 cDNA sequence identified encoded a $28-\mathrm{kD}$ protein lacking the $\mathrm{N}$-terminal mitochondrial targeting sequence (Yang et al., 2000). Several recent studies have identified a longer isoform of murine SIRT3 encoding a $37 \mathrm{kD}$ protein, however, that can be imported into mitochondria and processed into the mature $28 \mathrm{kD}$ protein (Cooper et al., 2009; Jin et al., 2009; Bao et al., 2010; Yang et al., 2010). Whether or not an active fraction of SIRT3 exists

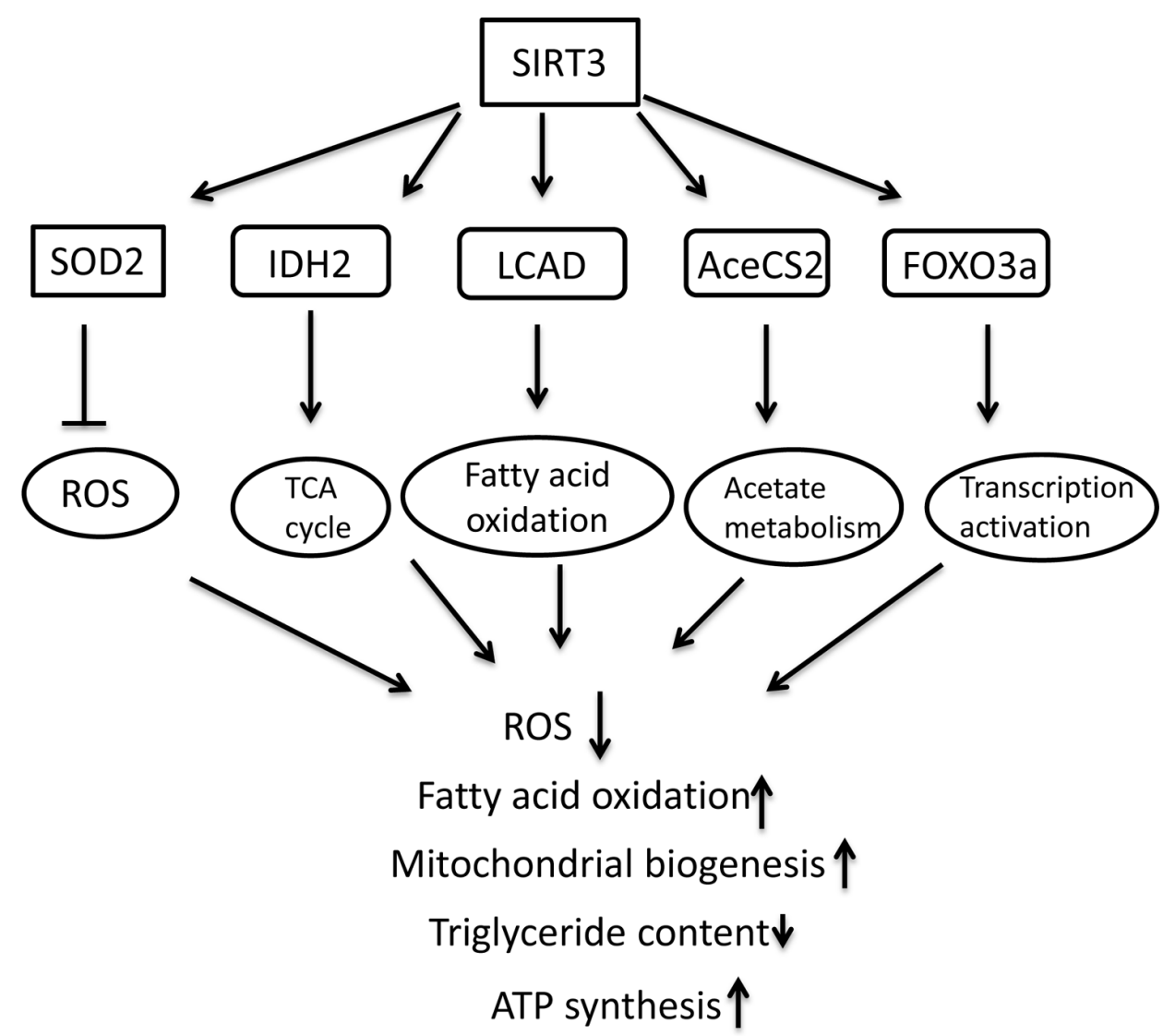

FIGURE 2 | Schematic overview of molecular targets of SIRT3 and the role in regulating metabolism in mitochondria. 
outside mitochondria and modifies extra-mitochondrial proteins remains controversial.

\section{SIRT3 AND METABOLIC HOMEOSTASIS}

Emerging data have shown that one major function of SIRT3 is regulation of mitochondrial electron transport chain activity to maintain energy homeostasis. The main energy source in mitochondria is pyruvate, a product of glycolysis. Alternatively, mitochondria also burn fatty acids, amino acids, and acetates when pyruvate is deficient. For fatty acid catabolism, long-chain acyl coenzyme A dehydrogenase (LCAD) is a key enzyme that breaks down fatty acids and generates acetyl-CoA, stimulating $\beta$-oxidation. In SIRT3 knockout mice, LCAD is hyperacetylated at Lys42, leading to decreases in enzymatic activity, $\beta$-oxidation, and ATP level (Hirschey et al., 2010). Interestingly, these mice do not tolerate cold exposure during fasting (Hirschey et al., 2010). SIRT3 directly deacetylates LACD at Lys42 and increases LACD activity (Hirschey et al., 2010). In addition, SIRT3 may promote $\beta$ oxidation via multiple mechanisms, such as by deacetylating other $\beta$-oxidation enzymes, including the short-chain L-3-hydroxyacylCoA dehydrogenase and the very-long-chain acyl coenzyme A dehydrogenase (Hallows et al., 2011), facilitating mitochondrial adaptation to fuel changes.

Glucose is the major energy source for cells. When its availability is limited, however, alternative fuels become increasingly important for cell survival. The first step of glycolysis is the conversion of glucose to glucose-6-phosphate, a reaction catalyzed by hexokinases. It is reported that SIRT3 deacetylates cyclophilin D (Hafner et al., 2010; Shulga et al., 2010), which leads to the dissociation of hexokinase II and mitochondria, decreases glucose metabolism, and stimulates oxidative phosphorylation (Shulga et al., 2010).

Acetate derived from acetic acid and alcohol is also used as a mitochondrial fuel, although this only occurs in extreme circumstances of nutrient depletion. In the initial step acetate is converted to acetyl-CoA catalyzed by acetyl-CoA synthetases. Acetyl-CoA synthetase 2 is the mitochondrial form of the enzyme. SIRT3 deacetylates acetyl-CoA synthetase 2 and enhances its activity, leading to increased production of acetyl-CoA (Hallows et al., 2006; Schwer et al., 2006). The acetyl-CoA from fatty acids and acetates as well as $\alpha$-ketoglutarate from amino acids can enter the Krebs cycle. These two reactions are enhanced by SIRT3 (Hallows et al., 2006; Schwer et al., 2006; Lombard et al., 2007; Schlicker et al., 2008). Additionally, SIRT3 directly stimulates the Krebs cycle. The third step of the cycle is the conversion of 6-carbon isocitrate to 5-carbon $\alpha$-ketoglutarate, a process catalyzed by isocitrate dehydrogenase 2 (IDH2). A recent study shows that SIRT3 directly deacetylates this dehydrogenase to increase its activity (Someya et al., 2010).

$\mathrm{NADH}$ dehydrogenase 1 alpha subcomplex subunit 9 (NDUFA9) is an enzyme of mitochondrial complex I that is acetylated at Lys370 (Kim et al., 2006). SIRT3 physically interacts with NDUFA9 and deacetylates it. SIRT3 knockout enhances its acetylation and reduces the activity of complex I (Ahn et al., 2008), indicating that SIRT3 is a positive regulator of complex I. Complex II, also known as succinate dehydrogenase, is composed of four subunits, including the flavoprotein succinate dehydrogenase subunit A (SdhA). In SIRT3 knockout mice, SdhA is hyperacetylated at several lysine residues, and shows decreased activity of complex II (Cimen et al., 2010). SIRT3 overexpression reverses the acetylation of SdhA and increases complex II activity (Cimen et al., 2010), indicating that SdhA is a SIRT3 substrate, and that SIRT3 is also a positive regulator of complex II.

SIRT3 is also reported to bind the alpha subunit 1 of the F1 particle of ATP synthase (Law et al., 2009), but the function is unclear. Taken together, these results suggest that SIRT3 promotes ATP generation through enhancing action of several enzymes involved in energy metabolism. Further supporting this role, SIRT3-knockout mice show substantial acetylation of mitochondrial proteins, and have reduced ATP levels at baseline and during cellular stress (Ahn et al., 2008).

Mitochondria are also the major sites for the generation of the ROS, superoxide, and also where the superoxide is dismuted by mitochondrial MnSOD. Recent reports show that SIRT3 deacetylates MnSOD at Lys122 and increases its activity, reducing oxidative and radiation stress in mice (Qiu et al., 2010; Tao et al., 2010). Overexpression of SIRT3 protects HEK293 from oxidative stress and prevents age-related cochlear cell death in mice (Someya et al., 2010). Overall, this suggests anti-oxidative and neuroprotective roles of SIRT3. New data suggest that the SIRT3 deacetylase plays a key role in bolstering mitochondrial anti-oxidant defenses during CR (Qiu et al., 2010; Someya et al., 2010).

In future studies, it will be of interest to define the mechanism whereby acetylation of electron transport chain subunits affects generation of ATP. It is also important to elucidate why it might be desirable under some physiologic conditions to downregulate electron transport chain activity by increased acetylation. As an added wrinkle, SIRT3 negatively regulates translation within mitochondria by deacetylating the ribosomal protein MRPL10, a function proposed to reduce respiration (Yang et al., 2010).

SIRT3 levels are increased in adipose tissue, skeletal muscle, and liver during CR (Shi et al., 2005; Palacios et al., 2009; Schwer et al., 2009; Hirschey et al., 2010), and conversely decline in response to high-fat feeding (Palacios et al., 2009; Bao et al., 2010; Kendrick et al., 2011). These expression data suggest that SIRT3 might play a role in the response to metabolic homeostasis. In mammals, two independent studies showed that SIRT3 interacts with and deacetylates acetyl-CoA synthetase 2 (AceCS2) at the active site lysine to promote AceCS2 activity (Hallows et al., 2006; Schwer et al., 2006). Under fed conditions, the majority of acetyl-CoA is generated through metabolism of pyruvate by Pyruvate dehydrogenase complex (PDC) and by fatty acid $\beta$ oxidation, largely bypassing the need for AceCS2. In this regard, studies of AceCS2-deficient mice revealed that AceCS2 is specifically required for metabolic homeostasis when the mice are fed a low carbohydrate/high fat diet (LC/HFD); AceCS2-deficient animals are essentially normal on a chow diet but show poor weight gain, hypothermia, hypoglycemia, and impaired survival on an LC/HFD (Sakakibara et al., 2009). Presumably the role of SIRT3 in regulating AceCS2 could also be important during fasting, when acetate can be used as a source of energy in extrahepatic tissues (Hirschey et al., 2010). Indeed, SIRT3 has recently been shown to deacetylate and activate 3-hydroxy-3-methylglutarylCoA synthase 2 (HMGCS2), a mitochondrial enzyme that converts 
acetyl-CoA into ketone bodies (acetoacetate, $\beta$-hydroxybutyrate, and acetone) in the liver under fasting conditions, which can in turn be used as a source of energy in certain tissues including the brain (Shimazu et al., 2010). SIRT3-deficient mice are unable to produce normal levels of ketone bodies upon fasting.

\section{SIRT, LIFESPAN AND AGE-ASSOCIATED PHENOTYPES}

The role of SIRT3 in aging is of considerable interest because it appears to suppress ROS, one of the causes contributing to the process of aging. In addition to elucidating its roles in regulating specific biochemical pathways in mitochondria, there is great interest in testing whether SIRT3 might modulate age-associated phenotypes, or indeed lifespan itself. In this regard, some studies have linked polymorphisms in the SIRT3 genomic locus to human longevity, although others have failed to demonstrate this association (Rose et al., 2003; Bellizzi et al., 2005, 2007; Lescai et al., 2009). A polymorphism associated with decreased SIRT3 mRNA expression was present in cohorts of young but not old men, suggesting that reduced SIRT3 expression may be detrimental to survival in old age (Bellizzi et al., 2005, 2009). In sedentary individuals, SIRT3 protein expression declined with age in skeletal muscle mitochondria, concomitant with a reduction in respiratory function (Lanza et al., 2008).

Age-related hearing loss (ARHL) is a common problem in the elderly, occurring secondary to cell loss and other degenerative changes in the cochlea. An elegant study has firmly established a role for SIRT3 in preventing ARHL (Someya et al., 2010). One mechanism by which SIRT3 mediates this effect is by deacetylation of IDH2 (Schlicker et al., 2008; Someya et al., 2010), which converts isocitrate to $\alpha$-ketoglutarate concomitant with reduction of $\mathrm{NADP}^{+}$. NADPH in turn allows regeneration of reduced glutathione to promote mitochondrial oxidative defense. In response to $\mathrm{CR}$, wild-type mice, but not SIRT3-deficient animals, show increased NADPH levels, increased reduced glutathione in mitochondria, and decreased DNA damage in the cochlea and in other tissues. In tissue culture cells, overexpression of SIRT3 or IDH2 protects against oxidative stress-induced cell death, and the two proteins together have a synergistic pro-survival effect. These results do not rule out the possibility that SIRT3 might modify other substrates in addition to IDH2 to prevent AHRL during CR. Similarly, Qiu et al. (2010) reported that SIRT3-deficient mice fail to suppress ROS levels and macromolecular damage during CR. They find that SIRT3 directly deacetylates SOD2 to increase its activity during CR, whereas SIRT3-deficient mice do not show SOD2 deacetylation in response to this diet (Qiu et al., 2010). Overall, these papers point to crucial role for SIRT3 in suppressing oxidative damage and its negative sequel during CR. It remains to be seen how SIRT3, or the other mitochondrial sirtuins, might affect other phenotypes of aging and/or effects of CR. The reduction of serum insulin and triglycerides that normally occurs during CR is not observed in SIRT3-deficient mice (Someya et al., 2010), implying that SIRT3 plays additional, uncharacterized roles in the adaptation to this dietary regimen. A recent study indicates that upregulation of SIRT3 indeed reverses aging-associated degeneration in hematopoietic stem cells (Brown et al., 2013), and SIRT3 may promote organismal longevity by maintaining the integrity of tissue-specific stem cells.

\section{SIRT3 AND MITOCHONDRIAL PROTEIN ACETYLATION: UNRESOLVED QUESTIONS}

Acetylation of mitochondrial proteins plays a major role in regulating functions of this organelle. Despite the rapid progress in this area, there are still many outstanding questions remain that will no doubt provide fruitful avenues for research for years to come. In particular, how mitochondrial proteins are acetylated in the first place is currently unknown. The identity of putative mitochondrial acetyltransferases remains elusive; identification of such proteins would represent a major step forward in this field. Alternatively, or in addition to enzymatic acetylation within mitochondria, mitochondrial proteins could in principle be acetylated outside this organelle, before or concomitant with mitochondrial import or even be acetylated non-enzymatically. These latter models would not permit rapid cycles of acetylation/deacetylation of mitochondrial proteins to regulate target protein function in response to varied environmental challenges. Instead, after deacetylation, restoration of acetylation status would require new protein synthesis. Such models could be distinguished through pulse-chase experiments assessing acetylation of newly synthesized mitochondrial proteins before and after mitochondrial import.

Similarly, how SIRT3 activity is regulated in the mitochondria is incompletely understood. SIRT3 requires $\mathrm{NAD}^{+}$, and therefore mitochondrial $\mathrm{NAD}^{+}$levels play a critically important role in governing mitochondrial SIRT3 function. Increased NADH generation from $\mathrm{NAD}^{+}$that occurs with HFD and leads to reduced SIRT3 function may explain the increased global mitochondrial protein acetylation observed during this diet, as could increase levels of acetyl-CoA, the substrate for acetyltransferases (Kendrick et al., 2011). This overall increased acetylation may represent the net effect of increased acetyltransferase activity superimposed upon elevated SIRT3 function; alternatively, some protein species hyperacetylated during CR or other conditions may not be substrates for mitochondrial SIRT3. The activity of SIRT3 and other mitochondrial sirtuins might be influenced by other conditions in addition to $\mathrm{NAD}^{+}$levels, such as post-translational modification or interactions with regulatory proteins.

It is currently unclear whether interventions that have an impact on acetylation of many mitochondrial proteins - SIRT3 deficiency, such as CR, HFD - lead to modification of common sets of proteins on the same lysine sites, or whether this response is tailored to different environmental perturbations. Similarly, it remains unclear whether the mitochondrial sirtuins share common targets and/or functions in common. This question could be addressed in mice or cells with mitochondrial sirtuin deficiencies or knockdowns. Given that SIRT3 deacetylates many proteins in mitochondria as well as suppresses some age-associated phenotypes, it will be of interest to test whether acetylation of mitochondrial proteins changes with age, either individually or globally, and whether prevention of this effect might have a beneficial effect on health span or even lifespan.

In addition, whereas the functional impact of acetylation on a few individual protein targets is clear, a global understanding of how SIRT3 affects the activity of metabolic pathways in mitochondria, cells, tissues, and the organism overall is still lacking. Answers to these and related questions involving SIRT3 
and mitochondrial protein acetylation will no doubt reveal novel aspects of mitochondrial biology, and perhaps ultimately provide the basis for novel therapeutic strategies for a variety of disorders.

\section{FURTHER PERSPECTIVES}

In the past decade, the function of mammalian sirtuins has been investigated in greater detail than ever before, and we now have a much better molecular understanding of the multiple roles this unique family of enzymes plays in seemingly every biological process. There is little doubt that sirtuins have emerged as critical modulators of metabolic adaptive responses, and their activities have been linked to metabolic abnormalities as well as

\section{REFERENCES}

Adler, A. S., Sinha, S., Kawahara, T. L., Zhang, J. Y., Segal, E., and Chang, H. Y. (2007). Motif module map reveals enforcement of aging by continual NF-kappaB activity. Genes Dev. 21, 3244-3257. doi: 10.1101/gad.1588507

Ahn, B. H., Kim, H. S., Song, S., Lee, I. H., Liu, J., Vassilopoulos, A., et al. (2008). A role for the mitochondrial deacetylase Sirt3 in regulating energy homeostasis. Proc. Natl. Acad. Sci. U.S.A. 105, 14447-14452. doi: 10.1073/pnas.0803790105

Albani, D., Polito, L., Batelli, S., De Mauro, S., Fracasso, C., Martelli, G., et al. (2009). The SIRT1 activator resveratrol protects SK-N-BE cells from oxidative stress and against toxicity caused by alpha-synuclein or amyloid-beta (1-42) peptide. J. Neurochem. 110, 1445-1456. doi: 10.1111/j.1471-4159.2009.06228.x

Alvira, D., Yeste-Velasco, M., Folch, J., Verdaguer, E., Canudas, A. M., Pallas, M., et al. (2007). Comparative analysis of the effects of resveratrol in two apoptotic models: inhibition of complex I and potassium deprivation in cerebellar neurons. Neuroscience 147, 746-756. doi: $\quad 10.1016 /$ j.neuroscience.2007. 04.029

Araki, T., Sasaki, Y., and Milbrandt, J. (2004). Increased nuclear NAD biosynthesis and SIRT1 activation prevent axonal degeneration. Science 305, 1010-1013. doi: 10.1126/science. 1098014

Babetto, E., Beirowski, B., Janeckova, L., Brown, R., Gilley, J., Thomson, D., et al. (2010). Targeting NMNAT1 to axons and synapses transforms its neuroprotective potency in vivo. J. Neurosci. 30, 13291-13304. doi: 10.1523/JNEUROSCI.1189-10.2010

Bao, J., Scott, I., Lu, Z., Pang, L., Dimond, C. C., Gius, D., et al. (2010). SIRT3 is regulated by nutrient excess and modulates hepatic susceptibility to lipotoxicity. Free
Radic. Biol. Med. 49, 1230-1237. doi: 10.1016/j.freeradbiomed.2010. 07.009

Baur, J. A., Pearson, K. J., Price, N. L., Jamieson, H. A., Lerin, C., Kalra A., et al. (2006). Resveratrol improves health and survival of mice on a highcalorie diet. Nature 444, 337-342. doi: 10.1038/nature05354

Bellizzi, D., Covello, G., Di Cianni, F., Tong, Q., and De Benedictis, G. (2009). Identification of GATA2 and AP-1 activator elements within the enhancer VNTR occurring in intron 5 of the human SIRT3 gene. Mol. Cells 28, 87-92. doi: 10.1007/s10059-0090110-3

Bellizzi, D., Dato, S., Cavalcante, P., Covello, G., Di Cianni, F., Passarino, G., et al. (2007). Characterization of a bidirectional promoter shared between two human genes related to aging: SIRT3 and PSMD13. Genomics 89, 143-150. doi: 10.1016/j.ygeno.2006.09.004

Bellizzi, D., Rose, G., Cavalcante, P., Covello, G., Dato, S., De Rango, F., etal. (2005). A novel VNTR enhancer within the SIRT3 gene, a human homologue of SIR2, is associated with survival at oldest ages. Genomics 85, 258-263. doi: 10.1016/ j.ygeno.2004.11.003

Biessels, G. J., and Kappelle, L. J. (2005). Increased risk of Alzheimer's disease in Type II diabetes: insulin resistance of the brain or insulin-induced amyloid pathology? Biochem. Soc. Trans. 33, 1041-1044. doi: 10.1042/ BST20051041

Blum, C. A., Ellis, J. L., Loh, C., Ng, P. Y., Perni, R. B., and Stein, R. L. (2011). SIRT1 modulation as a novel approach to the treatment of diseases of aging. J. Med. Chem. 54, 417-432. doi: 10.1021/jm100861p

Boveris, A., and Navarro, A. (2008). Brain mitochondrial dysfunction in aging. IUBMB Life 60, 308-314. doi: 10.1002/iub.46

Braunstein, M., Rose, A. B., Holmes, S. G., Allis, C. D., and Broach, J.

age-associated neurodegeneration. Yet, key questions will keep investigators busy in the coming years. We still have poor understanding of the molecular mechanisms regulating expression and activity of the sirtuins, and of the precise stimuli that regulate these proteins, and whether the activities of different sirtuins are regulated in a coordinated fashion. In other words, is there cross-talk between sirtuins? Will future studies cement the argument that sirtuins are, indeed, critical modulators of lifespan? This review has focused on SIRT1 and SIRT3. Even less is known about other sirtuins. Further investigation into the targets and functions of this unique family of sirtuins will help develop new strategies for protection against and recovery from common aging-related neurological diseases and promote successful aging.

R. (1993). Transcriptional silencing in yeast is associated with reduced nucleosome acetylation. Genes Dev 7, 592-604. doi: 10.1101/gad.7.4.592

Brooks, C. L., and Gu, W. (2009). How does SIRT1 affect metabolism, senescence and cancer? Nat. Rev. Cancer 9, 123-128. doi: 10.1038/nrc2562

Brown, K., Xie, S., Qiu, X., Mohrin, M., Shin, J., Liu, Y., et al. (2013). SIRT3 reverses aging-associated degeneration. Cell Rep. 3, 319-327. doi: 10.1016/j.celrep.2013.01.005

Burnett, C., Valentini, S., Cabreiro, F., Goss, M., Somogyvari, M., Piper, M. D., et al. (2011). Absence of effects of Sir2 overexpression on lifespan in, $C$. elegans and Drosophila. Nature 477, 482-485. doi: 10.1038/nature10296

Campisi, J. (2005). Suppressing cancer: the importance of being senescent. Science 309, 886-887. doi: $10.1126 /$ science. 1116801

Canto, C., and Auwerx, J. (2009). Caloric restriction, SIRT1 and longevity. Trends Endocrinol. Metab. 20, 325-331. doi: 10.1016/j.tem. 2009.03.008

Chalkiadaki, A., and Guarente, L. (2012). Sirtuins mediate mammalian metabolic responses to nutrient availability. Nat. Rev. Endocrinol. 8, 287296. doi: 10.1038/nrendo.2011.225

Chao, J., Yu, M. S., Ho, Y. S., Wang, M., and Chang, R. C. (2008). Dietary oxyresveratrol prevents parkinsonian mimetic 6hydroxydopamine neurotoxicity. Free Radic. Biol. Med. 45, 1019-1026. doi: 10.1016/j.freeradbiomed.2008. 07.002

Chen, D., Steele, A. D., Lindquist, S., and Guarente, L. (2005a). Increase in activity during calorie restriction requires Sirt1. Science 310, 1641. doi: 10.1126/science. 1118357

Chen, J., Zhou, Y., Mueller-Steiner, S., Chen, L. F., Kwon, H., Yi, S., etal. (2005b). SIRT1 protects against microglia-dependent amyloid-beta toxicity through inhibiting NF-kappaB signaling.
J. Biol. Chem. 280, 40364-40374. doi: 10.1074/jbc.M509329200

Chen, L. F., and Greene, W. C. (2003). Regulation of distinct biological activities of the NF-kappaB transcription factor complex by acetylation. J. Mol. Med. (Berl.) 81, 549-57. doi: 10.1007/s00109-003-0469-0

Chong, Z. Z., Lin, S. H., Li, F., and Maiese, K. (2005). The sirtuin inhibitor nicotinamide enhances neuronal cell survival during acute anoxic injury through AKT, BAD, PARP, and mitochondrial associated "anti-apoptotic" pathways. Curr. Neurovasc. Res. 2, 271-285. doi: $10.2174 / 156720205774322584$

Chong, Z. Z., and Maiese, K. (2008). Enhanced tolerance against early and late apoptotic oxidative stress in mammalian neurons through nicotinamidase and sirtuin mediated pathways. Curr. Neurovasc. Res. 5, 159-170. doi: 10.2174/ 156720208785425666

Cimen, H., Han, M. J., Yang, Y., Tong, Q., Koc, H., and Koc, E. C. (2010). Regulation of succinate dehydrogenase activity by SIRT3 in mammalian mitochondria. Biochemistry 49, 304311. doi: 10.1021/bi901627u

Cooper, H. M., Huang, J. Y., Verdin, E., and Spelbrink, J. N. (2009). A new splice variant of the mouse SIRT3 gene encodes the mitochondrial precursor protein. PLoS ONE 4:e4986. doi: 10.1371/journal.pone.0004986

Dali-Youcef, N., Lagouge, M., Froelich, S., Koehl, C., Schoonjans, K., and Auwerx, J. (2007). Sirtuins: the 'magnificent seven', function, metabolism and longevity. Ann. Med. 39, 335-345. doi: 10.1080/ 07853890701408194

Della-Morte, D., Dave, K. R., DeFazio, R. A., Bao, Y. C., Raval, A. P., and Perez-Pinzon, M. A. (2009). Resveratrol pretreatment protects rat brain from cerebral ischemic damage via a sirtuin 1-uncoupling protein 2 pathway. Neuroscience 159, 993-1002. doi: 10.1016/j.neuroscience.2009.01.017 
Dietrich, M. O., Antunes, C., Geliang, G., Liu, Z. W., Borok, E., Nie, Y., et al. (2010). Agrp neurons mediate Sirtl's action on the melanocortin system and energy balance: roles for Sirt1 in neuronal firing and synaptic plasticity. J. Neurosci. 30, 11815-11825. doi: 10.1523/JNEUROSCI.223410.2010

Donmez, G., Arun, A., Chung, C. Y., McLean, P. J., Lindquist, S., and Guarente, L. (2012). SIRT1 protects against alpha-synuclein aggregation by activating molecular chaperones. J. Neurosci. 32, 124-132. doi: 10.1523/JNEUROSCI.3442-11.2012

Donmez, G., Wang, D., Cohen, D. E., and Guarente, L. (2010). SIRT1 suppresses beta-amyloid production by activating the alpha-secretase gene ADAM10. Cell 142, 320-332. doi: 10.1016/j.cell.2010.06.020

Drew, B., and Leeuwenburgh, C. (2004). Ageing and subcellular distribution of mitochondria: role of mitochondrial DNA deletions and energy production. Acta Physiol. Scand. 182, 333-341. doi: 10.1111/j.1365201X.2004.01371.x

Duan, W., Guo, Z., Jiang, H., Ware, M., Li, X. J., and Mattson, M. P. (2003). Dietary restriction normalizes glucose metabolism and BDNF levels, slows disease progression, and increases survival in huntingtin mutant mice. Proc. Natl. Acad. Sci. U.S.A. 100, 2911-2916. doi: 10.1073/ pnas.0536856100

Duan, W., and Mattson, M. P. (1999). Dietary restriction and 2-deoxyglucose administration improve behavioral outcome and reduce degeneration of dopaminergic neurons in models of Parkinson's disease. J. Neurosci. Res. 57, 195-206. doi: 10.1002/(SICI)10974547(19990715)57:2

Finley, L. W., and Haigis, M. C. (2009). The coordination of nuclear and mitochondrial communication during aging and calorie restriction. Ageing Res. Rev. 8, 173-188. doi: 10.1016/j.arr.2009.03.003

Guarente, L., and Franklin, H. (2011). Epstein lecture: sirtuins, aging, and medicine. N. Engl. J. Med. 364, 22352244. doi: 10.1056/NEJMra1 100831

Hafner, A. V., Dai, J., Gomes, A. P., Xiao, C. Y., Palmeira, C. M., Rosenzweig, A., et al. (2010). Regulation of the MPTP by SIRT3-mediated deacetylation of CypD at lysine 166 suppresses agerelated cardiac hypertrophy. Aging (Albany NY) 2, 914-923.

Haigis, M. C., and Guarente, L. P. (2006). Mammalian sirtuins emerging roles in physiology, aging, and calorie restriction.
Genes Dev. 20, 2913-2921. doi: 10.1101/gad.1467506

Hallows, W. C., Lee, S., and Denu, J. M. (2006). Sirtuins deacetylate and activate mammalian acetylCoA synthetases. Proc. Natl. Acad. Sci. U.S.A. 103, 10230-10235. doi: 10.1073/pnas.0604392103

Hallows, W. C., Yu, W., Smith, B. C., Devries, M. K., Ellinger, J. J., Someya, S., etal. (2011). Sirt3 promotes the urea cycle and fatty acid oxidation during dietary restriction. Mol. Cell. 41, 139-149. doi: 10.1016/j.molcel.2011.01.002

Hardie, D. G. (2011). AMP-activated protein kinase: an energy sensor that regulates all aspects of cell function. Genes Dev. 25, 1895-1908. doi: 10.1101/gad.17420111

Herranz, D., Munoz-Martin, M. Canamero, M., Mulero, F., MartinezPastor, B., Fernandez-Capetillo, O., et al. (2010). Sirtl improves healthy ageing and protects from metabolic syndrome-associated cancer. Nat. Commun. 1, 3. doi: 10.1038/ ncomms 1001

Hirschey, M. D., Shimazu, T., Goetzman, E., Jing, E., Schwer, B. Lombard, D. B., et al. (2010). SIRT3 regulates mitochondrial fattyacid oxidation by reversible enzyme deacetylation. Nature 464, 121-125. doi: $10.1038 /$ nature 08778

Howitz, K. T., Bitterman, K. J., Cohen, H. Y., Lamming, D. W., Lavu, S., Wood, J. G., et al. (2003). Small molecule activators of sirtuins extend Saccharomyces cerevisiae lifespan. Nature 425, 191-196. doi: 10.1038 /nature 01960

Hubbard, B. P., Gomes, A. P., Dai, H., Li, J., Case, A. W., Considine, T., et al. (2013). Evidence for a common mechanism of SIRT1 regulation by allosteric activators. Science 339, 1216-1219. doi: 10.1126/science. 1231097

Imai, S., and Guarente, L. (2010). Ten years of NAD-dependent SIR2 family deacetylases: implications for metabolic diseases. Trends Pharmacol. Sci. 31, 212-220. doi: 10.1016/j.tips.2010.02.003

Jeong, H., Cohen, D. E., Cui, L., Supinski, A., Savas, J. N., Mazzulli, J. R., etal. (2011). Sirtl mediates neuroprotection from mutant huntingtin by activation of the TORC1 and CREB transcriptional pathway. Nat. Med. 18, 159-165. doi: 10.1038/nm.2559

Jiang, J. C., Jaruga, E., Repnevskaya, M. V., and Jazwinski, S. M. (2000). An intervention resembling caloric restriction prolongs life span and retards aging in yeast. FASEB $J$.
14, 2135-2137. doi: 10.1096/fj.00 0242fje

Jiang, M., Wang, J., Fu, J., Du, L. Jeong, H., West, T., et al. (2011). Neuroprotective role of Sirt1 in mammalian models of Huntington's disease through activation of multiple Sirtl targets. Nat. Med. 18, 153-158. doi: $10.1038 / \mathrm{nm} .2558$

Jin, L., Galonek, H., Israelian, K., Choy, W., Morrison, M., Xia, Y., et al. (2009). Biochemical characterization, localization, and tissue distribution of the longer form of mouse SIRT3. Protein Sci. 18, 514-525. doi: $10.1002 /$ pro. 50

Julien, C., Tremblay, C., Emond, V., Lebbadi, M., Salem, N. Jr., Bennett, D. A., et al. (2009). Sirtuin 1 reduction parallels the accumulation of tau in Alzheimer disease. J. Neuropathol. Exp. Neurol. 68, 48-58. doi: 10.1097/NEN.0b013e3181922348

Jung, C. H., Ro, S. H., Cao, J., Otto, N. M., and Kim, D. H. (2010). mTOR regulation of autophagy. FEBS Lett. 584, 1287-1295. doi: 10.1016/j.febslet.2010.01.017

Kaeberlein, M., McVey, M., and Guarente, L. (1999). The SIR2/3/4 complex and SIR2 alone promote longevity in Saccharomyces cerevisiae by two different mechanisms. Genes Dev. 13, 2570-2580. doi: 10.1101/ gad.13.19.2570

Kakefuda, K., Fujita, Y., Oyagi, A., Hyakkoku, K., Kojima, T., Umemura, K., et al. (2009). Sirtuin 1 overexpression mice show a reference memory deficit, but not neuroprotection. Biochem. Biophys Res. Commun. 387, 784-788. doi: 10.1016/j.bbrc.2009.07.119

Kendrick, A. A., Choudhury, M., Rahman, S. M., McCurdy, C. E., Friederich, M., Van Hove, J. L., et al. (2011). Fatty liver is associated with reduced SIRT3 activity and mitochondrial protein hyperacetylation. Biochem. J. 433, 505-514. doi: 10.1042/BJ20100791

Kim, D., Nguyen, M. D., Dobbin, M. M., Fischer, A., Sananbenesi, F., Rodgers, J. T., et al. (2007). SIRT1 deacetylase protects against neurodegeneration in models for Alzheimer's disease and amyotrophic lateral sclerosis. EMBO J. 26, 3169-3179. doi: 10.1038/sj.emboj.7601758

Kim, S. C., Sprung, R., Chen, Y., Xu, Y., Ball, H., Pei, J., et al. (2006). Substrate and functional diversity of lysine acetylation revealed by a proteomics survey. Mol. Cell. 23, 607-618. doi: 10.1016/j.molcel.2006.06.026

Klar, A. J., and Fogel, S. (1979). Activation of mating type genes by transposition in Saccharomyces cerevisiae.
Proc. Natl. Acad. Sci. U.S.A. 76, 45394543. doi: 10.1073/pnas.76.9.4539

Koubova, J., and Guarente, L. (2003). How does calorie restriction work? Genes Dev. 17, 313-321. doi: 10.1101/gad.1052903

Lan, F., Cacicedo, J. M., Ruderman, N., and Ido, Y. (2008). SIRT1 modulation of the acetylation status, cytosolic localization, and activity of LKB1. Possible role in AMPactivated protein kinase activation. $J$. Biol. Chem. 283, 27628-27635. doi: 10.1074/jbc.M805711200

Lanza, I. R., Short, D. K., Short, K. R., Raghavakaimal, S., Basu, R., Joyner, M. J., et al. (2008). Endurance exercise as a countermeasure for aging. Diabetes 57, 2933-2942. doi: $10.2337 / \mathrm{db} 08-0349$

Lassmann, H. (2010). Axonal and neuronal pathology in multiple sclerosis: what have we learnt from animal models. Exp. Neurol. 225, 2-8. doi: 10.1016/j.expneurol.2009.10.009

Lavu, S., Boss, O., Elliott, P. J., and Lambert, P. D. (2008). Sirtuins - novel therapeutic targets to treat age-associated diseases. Nat. Rev. Drug Discov. 7, 841-853. doi: 10.1038/nrd2665

Law, I. K., Liu, L., Xu, A., Lam, K. S., Vanhoutte, P. M., Che, C. M., et al. (2009). Identification and characterization of proteins interacting with SIRT1 and SIRT3: implications in the anti-aging and metabolic effects of sirtuins. Proteomics 9, 2444-2456. doi: 10.1002/pmic. 200800738

Lee, I. H., Cao, L., Mostoslavsky, R., Lombard, D. B., Liu, J., Bruns, N. E., et al. (2008). A role for the NADdependent deacetylase Sirtl in the regulation of autophagy. Proc. Natl. Acad. Sci. U.S.A. 105, 3374-3379. doi: 10.1073/pnas.0712145105

Lescai, F., Blanche, H., Nebel, A., Beekman, M., Sahbatou, M., Flachsbart, F., et al. (2009). Human longevity and 11p15.5: a study in 1321 centenarians. Eur. J. Hum. Genet. 17, 15151519. doi: 10.1038/ejhg.2009.54

Liu, D., Gharavi, R., Pitta, M., Gleichmann, M., and Mattson, M. P. (2009). Nicotinamide prevents $\mathrm{NAD}^{+}$depletion and protects neurons against excitotoxicity and cerebral ischemia: $\mathrm{NAD}^{+}$consumption by SIRT1 may endanger energetically compromised neurons. Neuromol. Med. 11, 28-42. doi: 10.1007/s12017-009-8058-1

Lombard, D. B., Alt, F. W., Cheng, H. L., Bunkenborg, J., Streeper, R. S., Mostoslavsky, R., et al. (2007). Mammalian Sir2 homolog SIRT3 regulates global mitochondrial lysine acetylation. Mol. Cell. Biol. 27, 8807-8814. doi: 10.1128/MCB.01636-07 
Luo, J., Nikolaev, A. Y., Imai, S., Chen, D., Su, F., Shiloh, A., et al. (2001). Negative control of p53 by Sir2alpha promotes cell survival under stress. Cell 107, 137-148. doi: 10.1016/S0092-8674(01)00524-4

Madeo, F., Tavernarakis, N., and Kroemer, G. (2010). Can autophagy promote longevity? Nat. Cell Biol. 12, 842-846. doi: 10.1038/ncb0910-842

Markert, C. D., Kim, E., Gifondorwa D. J., Childers, M. K., and Milligan, C. E. (2010). A single-dose resveratrol treatment in a mouse model of amyotrophic lateral sclerosis. J. Med. Food 13, 1081-1085. doi: 10.1089/jmf.2009.0243

Masoro, E. J. (2000). Caloric restriction and aging: an update. Exp. Gerontol. 35, 299-305. doi: 10.1016/S05315565(00)00084-X

Mihaylova, M. M., and Shaw, R. J. (2011). The AMPK signalling pathway coordinates cell growth, autophagy and metabolism. Nat. Cell Biol. 13, 1016-1023. doi: 10.1038/ ncb2329

Min, S. W., Cho, S. H., Zhou, Y., Schroeder, S., Haroutunian, V., Seeley, W. W., et al. (2010). Acetylation of tau inhibits its degradation and contributes to tauopathy. Neuron 67, 953-966. doi: 10.1016/ j.neuron.2010.08.044

Morris, K. C., Lin, H. W., Thompson, J. W., and Perez-Pinzon, M. A. (2011). Pathways for ischemic cytoprotection: role of sirtuins in caloric restriction, resveratrol, and ischemic preconditioning. J. Cereb. Blood Flow Metab. 31, 1003-1019. doi: $10.1038 /$ jcbfm.2010.229

Navarro, A., and Boveris, A. (2007). The mitochondrial energy transduction system and the aging process. Am. J. Physiol. Cell Physiol. 292, C670-C686. doi: 10.1152/ajpcell.00213.2006

Nemoto, S., Fergusson, M. M., and Finkel, T. (2005). SIRT1 functionally interacts with the metabolic regulator and transcriptional coactivator PGC1 alpha\}. J. Biol. Chem. 280, 1645616460. doi: 10.1074/jbc.M501485200

Nisoli, E., Tonello, C., Cardile, A., Cozzi, V., Bracale, R., Tedesco, L., et al. (2005). Calorie restriction promotes mitochondrial biogenesis by inducing the expression of eNOS. Science 310, 314-317. doi: 10.1126/science. 1117728

Okawara, M., Katsuki, H., Kurimoto, E., Shibata, H., Kume, T., and Akaike, A. (2007). Resveratrol protects dopaminergic neurons in midbrain slice culture from multiple insults. Biochem. Pharmacol. 73, 550 560. doi: 10.1016/j.bcp.2006.11.003
Onyango, P., Celic, I., McCaffery, J. M., Boeke, J. D., and Feinberg, A. P. (2002). SIRT3, a human SIR2 homologue, is an NAD-dependent deacetylase localized to mitochondria. Proc Natl. Acad. Sci. U.S.A. 99, $13653-$ 13658. doi: 10.1073/pnas.222538099 Palacios, O. M., Carmona, J. J., Michan, S., Chen, K. Y., Manabe, Y., Ward, J. L. III, et al. (2009). Diet and exercise signals regulate SIRT3 and activate AMPK and PGC-1alpha in skeletal muscle. Aging (Albany NY) 1, 771-783.

Pallas, M., Pizarro, J. G., GutierrezCuesta, J., Crespo-Biel, N., Alvira, D., Tajes, M., et al. (2008). Modulation of SIRT1 expression in different neurodegenerative models and human pathologies. Neuroscience 154, 1388-1397. doi: 10.1016/ j.neuroscience.2008.04.065

Pallos, J., Bodai, L., Lukacsovich, T., Purcell, J. M., Steffan, J. S., Thompson, L. M., et al. (2008). Inhibition of specific HDACs and sirtuins suppresses pathogenesis in a Drosophila model of Huntington's disease. Hum. Mol. Genet. 17, 3767-3775. doi: 10.1093/hmg/ddn273

Parker, J. A., Arango, M., Abderrahmane, S., Lambert, E., Tourette, C. Catoire, H., et al. (2005). Resveratrol rescues mutant polyglutamine cytotoxicity in nematode and mammalian neurons. Nat. Genet. 37, 349-350. doi: $10.1038 /$ ng1534

Patel, N. V., Gordon, M. N., Connor, K. E., Good, R. A., Engelman, R. W., Mason, J., etal. (2005). Caloric restriction attenuates Abeta-deposition in Alzheimer transgenic models. Neurobiol. Aging. 26, 995-1000. doi: 10.1016/ j.neurobiolaging.2004.09.014

Perry, V. H., Lunn, E. R., Brown, M. C., Cahusac, S., and Gordon, S. (1990). Evidence that the Rate of Wallerian degeneration is controlled by a single autosomal dominant gene. Eur. J. Neurosci. 2, 408-413. doi: 10.1111/j.1460-9568.1990.tb00433.x

Petersen, K. F., Befroy, D., Dufour, S., Dziura, J., Ariyan, C., Rothman, D. L., et al. (2003). Mitochondrial dysfunction in the elderly: possible role in insulin resistance. Science 300 , 1140-1142. doi: 10.1126/science. 1082889

Picard, F., Kurtev, M., Chung, N., Topark-Ngarm, A., Senawong, T., Machado De Oliveira, R., etal. (2004). Sirtl promotes fat mobilization in white adipocytes by repressing PPAR-gamma. Nature 429, 771-776. doi: 10.1038/nature02583

Puigserver, P., Wu, Z., Park, C. W., Graves, R., Wright, M., and
Spiegelman, B. M. (1998). A coldinducible coactivator of nuclear receptors linked to adaptive thermogenesis. Cell 92, 829-839. doi: 10.1016/S0092-8674(00)81410-5

Qin, W., Chachich, M., Lane, M., Roth, G., Bryant, M., de Cabo, R., et al. (2006a). Calorie restriction attenuates Alzheimer's disease type brain amyloidosis in Squirrel monkeys (Saimiri sciureus). J. Alzheimers Dis. 10, 417-422.

Qin, W., Yang, T., Ho, L., Zhao, Z., Wang, J., Chen, L., et al. (2006b). Neuronal SIRT1 activation as a novel mechanism underlying the prevention of Alzheimer disease amyloid neuropathology by calorie restriction. J. Biol. Chem. 281, 2174521754. doi: 10.1074/jbc.M602909200

Qiu, X., Brown, K., Hirschey, M. D. Verdin, E., and Chen, D. (2010). Calorie restriction reduces oxidative stress by SIRT3-mediated SOD2 activation. Cell Metab. 12, 662-667. doi 10.1016/j.cmet.2010.11.015

Ramadori, G., Lee, C. E., Bookout, A. L., Lee, S., Williams, K W., Anderson, J., et al. (2008) Brain SIRT 1: anatomical distribution and regulation by energy availability. J. Neurosci. 28, 9989-9996. doi 10.1523/JNEUROSCI.3257-08.2008

Raval, A. P., Dave, K. R., and PerezPinzon, M. A. (2006). Resveratrol mimics ischemic preconditioning in the brain. J. Cereb. Blood Flow Metab. 26, 1141-1147. doi: 10.1038/sj.jcbfm.9600262

Rodgers, J. T., Lerin, C., Haas, W., Gygi, S. P., Spiegelman, B. M. and Puigserver, P. (2005). Nutrient control of glucose homeostasis through a complex of PGC-1alpha and SIRT1. Nature 434, 113-118. doi: 10.1038/nature03354

Rogina, B., and Helfand, S. L. (2004). Sir2 mediates longevity in the fly through a pathway related to calorie restriction. Proc. Natl. Acad. Sci. U.S.A. 101, 15998-16003. doi 10.1073/pnas.0404184101

Rose, G., Dato, S., Altomare, K., Bellizzi, D., Garasto, S., Greco, V., et al. (2003). Variability of the SIRT3 gene, human silent information regulator Sir2 homologue, and survivorship in the elderly. Exp. Gerontol. $38,1065-1070$. doi: 10.1016/S05315565(03)00209-2

Rosen, D. R. (1993). Mutations in $\mathrm{Cu} / \mathrm{Zn}$ superoxide dismutase gene are associated with familial amyotrophic lateral sclerosis. Nature 364, 362. doi: $10.1038 / 364362 \mathrm{c} 0$

Sakakibara, I., Fujino, T., Ishii, M., Tanaka, T., Shimosawa, T., Miura, S., et al. (2009). Fasting-induced hypothermia and reduced energy production in mice lacking acetylCoA synthetase 2. Cell Metab. 9, 191202. doi: 10.1016/j.cmet.2008.12.008 Salminen, A., and Kaarniranta, K. (2012). AMP-activated protein kinase (AMPK) controls the aging process via an integrated signaling network. Ageing Res. Rev. 11, 230-241. doi: 10.1016/j.arr.2011. 12.005

Salminen, A., Kauppinen, A., Suuronen, T., and Kaarniranta, K. (2008a). SIRT1 longevity factor suppresses NF-kappaB-driven immune responses: regulation of aging via NF-kappaB acetylation? Bioessays 30, 939-942. doi: 10.1002/bies.20799

Salminen, A., Ojala, J., Huuskonen, J., Kauppinen, A., Suuronen, T., and Kaarniranta, K. (2008b). Interaction of aging-associated signaling cascades: inhibition of NF-kappaB signaling by longevity factors FoxOs and SIRT1. Cell. Mol. Life Sci. 65, 1049-1058. doi: 10.1007/s00018008-7461-3

Sasaki, Y., Vohra, B. P., Baloh, R. H., and Milbrandt, J. (2009). Transgenic mice expressing the Nmnat protein manifest robust delay in axonal degeneration in vivo. J. Neurosci. 29, 6526-6534. doi: 10.1523/ JNEUROSCI.1429-09.2009

Satoh, A., Brace, C. S., Ben-Josef, G., West, T., Wozniak, D. F., Holtzman, D. M., etal. (2010). SIRT1 promotes the central adaptive response to diet restriction through activation of the dorsomedial and lateral nuclei of the hypothalamus. J. Neurosci. 30, 10220-10232. doi: 10.1523/JNEUROSCI.1385-10.2010

Sauve, A. A. (2009). Pharmaceutical strategies for activating sirtuins. Curr. Pharm. Des. 15, 45-56. doi: 10.2174/138161209787185797

Sauve, A. A., Wolberger, C., Schramm, V. L., and Boeke, J. D. (2006). The biochemistry of sirtuins. Annu. Rev. Biochem. 75, 435-465. doi: 10.1146/ annurev.biochem.74.082803.133500

Schlicker, C., Gertz, M., Papatheodorou, P., Kachholz, B., Becker, C. F., and Steegborn, C. (2008). Substrates and regulation mechanisms for the human mitochondrial sirtuins Sirt3 and Sirt5. J. Mol. Biol. 382, 790-801. doi: 10.1016/j.jmb.2008. 07.048

Schmitz, M. L., Mattioli, I., Buss, H., and Kracht, M. (2004). NF-kappaB: a multifaceted transcription factor regulated at several levels. Chembiochem 5, 1348-1358. doi: 10.1002/cbic.200400144

Schwer, B., Bunkenborg, J., Verdin, R. O., Andersen, J. S., and Verdin, 
E. (2006). Reversible lysine acetylation controls the activity of the mitochondrial enzyme acetyl-CoA synthetase 2. Proc. Natl. Acad. Sci. U.S.A. 103, 10224-10229. doi: 10.1073/pnas.0603968103

Schwer, B., Eckersdorff, M., Li, Y., Silva, J. C., Fermin, D., Kurtev, M. V., et al. (2009). Calorie restriction alters mitochondrial protein acetylation. Aging Cell 8, 604-606. doi: 10.1111/j.1474-9726.2009.00503.x

Schwer, B., North, B. J., Frye, R A., Ott, M., and Verdin, E. (2002). The human silent information regulator (Sir)2 homologue hSIRT3 is a mitochondrial nicotinamide adenine dinucleotide-dependent deacetylase. J. Cell Biol. 158, 647-657. doi: 10.1083/jcb.200205057

Shi, T., Wang, F., Stieren, E., and Tong, Q. (2005). SIRT3, a mitochondrial sirtuin deacetylase, regulates mitochondrial function and thermogenesis in brown adipocytes. J. Biol. Chem. 280, 13560-13567. doi: 10.1074/jbc.M414670200

Shimazu, T., Hirschey, M. D., Huang, J. Y., Ho, L. T., and Verdin, E. (2010). Acetate metabolism and aging: an emerging connection. Mech. Ageing Dev. 131, 511-516. doi: 10.1016/j.mad.2010.05.001

Shimokawa, I., and Trindade, L. S (2010). Dietary restriction and aging in rodents: a current view on its molecular mechanisms. Aging Dis. 1, 89-107.

Shindler, K. S., Ventura, E., Dutt, M., Elliott, P., Fitzgerald, D. C., and Rostami, A. (2010). Oral resveratrol reduces neuronal damage in a model of multiple sclerosis. $J$. Neuroophthalmol. 30, 328-339. doi: 10.1097/WNO.0b013e3181f7f833

Shindler, K. S., Ventura, E., Rex, T. S., Elliott, P., and Rostami, A. (2007). SIRT1 activation confers neuroprotection in experimental optic neuritis. Invest. Ophthalmol. Vis. Sci. 48, 3602-3609. doi: 10.1167/iovs.070131

Shulga, N., Wilson-Smith, R., and Pastorino, J. G. (2010). Sirtuin3 deacetylation of cyclophilin D induces dissociation of hexokinase II from the mitochondria. J. Cell Sci. 123, 894-902. doi: $10.1242 /$ jcs.061846

Sinclair, D. A. (2002). Paradigms and pitfalls of yeast longevity research. Mech. Ageing Dev. 123, 857-867. doi: 10.1016/S0047-6374(02)00023-4

Singh, K. K. (2004). Mitochondrial dysfunction is a common phenotype in aging and cancer. Ann. N. Y. Acad. Sci. 1019, 260-264. doi 10.1196/annals. 1297.043

Someya, S., Yu, W., Hallows, W. C., Xu, J., Vann, J. M., Leeuwenburgh, C. et al. (2010). Sirt3 mediates reduction of oxidative damage and prevention of age-related hearing loss under caloric restriction. Cell 143, 802-812. doi: 10.1016/j.cell.2010.10.002

Swerdlow, R. H. (2007). Treating neurodegeneration by modifying mitochondria: potential solutions to a "complex" problem. Antioxid. Redox Signal. 9, 1591-1603. doi: 10.1089/ars.2007.1676

Swerdlow, R. H. (2011). Brain aging, Alzheimer's disease, and mitochondria. Biochim. Biophys. Acta 1812, 1630-1639. doi: 10.1016/ j.bbadis.2011.08.012

Tang, B. L. (2009). Sirtl's complex roles in neuroprotection. Cell. Mol. Neurobiol. 29, 1093-1103. doi: 10.1007/s10571-009-9414-2

Tao, R., Coleman, M. C., Pennington, J. D., Ozden, O., Park, S. H., Jiang, H., etal. (2010). Sirt3mediated deacetylation of evolutionarily conserved lysine 122 regulates MnSOD activity in response to stress. Mol. Cell. 40, 893-904. doi: 10.1016/j.molcel.2010.12.013

Tissenbaum, H. A., and Guarente, L. (2001). Increased dosage of a sir-2 gene extends lifespan in Caenorhabditis elegans. Nature 410, 227-230. doi: 10.1038/35065638

Viswanathan, M., and Guarente, L. (2011). Regulation of Caenorhabditis elegans lifespan by sir-2.1 transgenes. Nature 477, E1-E2. doi: 10.1038 /nature 10440

Wakeling, L. A., Ions, L. J., and Ford, D. (2009). Could Sirtl-mediated epigenetic effects contribute to the longevity response to dietary restriction and be mimicked by other dietary interventions? Age (Dordr.) 31, 327-341. doi: 10.1007/s11357009-9104-5

Wang, J., Fivecoat, H., Ho, L., Pan, Y., Ling, E., and Pasinetti, G. M. (2010). The role of Sirtl: at the crossroad between promotion of longevity and protection against Alzheimer's disease neuropathology. Biochim. Biophys. Acta 1804, 1690 1694. doi: 10.1016/j.bbapap.2009. 11.015

Wang, J., Ho, L., Qin, W., Rocher, A. B., Seror, I., Humala, N., et al. (2005a). Caloric restriction attenuates beta-amyloid neuropathology in a mouse model of Alzheimer's disease. FASEB J. 19, 659-661. doi: 10.1096/fj.04-3182fje

Wang, J., Zhai, Q., Chen, Y., Lin, E., Gu, W., McBurney, M. W., et al. (2005b). A local mechanism mediates NAD-dependent protection of axon degeneration. J. Cell Biol. 170, 349-355. doi: 10.1083/jcb.200504028

Wang, P., Xu, T. Y., Guan, Y. F., Tian, W. W., Viollet, B., Rui, Y. C., et al. (2011) Nicotinamide phosphoribosyltransferase protects against ischemic stroke through SIRT1-dependent adenosine monophosphate-activated kinase pathway. Ann. Neurol. 69, 360-374. doi: 10.1002/ana.22236

Wang, S., Xing, Z., Vosler, P. S., Yin, H., Li, W., Zhang, F., et al. (2008). Cellular NAD replenishment confers marked neuroprotection against ischemic cell death: role of enhanced DNA repair. Stroke 39, 2587-2595. doi: 10.1161/ STROKEAHA.107.509158

Wareski, P., Vaarmann, A., Choubey, V., Safiulina, D., Liiv, J., Kuum, M., et al. (2009). PGC-1 \{alpha\} and PGC-1\{beta\} regulate mitochondrial density in neurons. J. Biol. Chem. 284, 21379-21385. doi: 10.1074/ jbc.M109.018911

Wood, J. G., Rogina, B., Lavu, S., Howitz, K., Helfand, S. L., Tatar, M., et al. (2004). Sirtuin activators mimic caloric restriction and delay ageing in metazoans. Nature 430, 686-689. doi: 10.1038 /nature02789

Xiong, S., Salazar, G., Patrushev, N., and Alexander, R. W. (2011). FoxO1 mediates an autofeedback loop regulating SIRT1 expression. J. Biol. Chem. 286, 5289-5299. doi: 10.1074/jbc.M110.163667

Yang, Y., Hubbard, B. P., Sinclair, D. A., and Tong, Q. (2010). Characterization of murine SIRT3 transcript variants and corresponding protein products. J. Cell. Biochem. 111, 1051-1058. doi: 10.1002/jcb. 22795

Yang, Y. H., Chen, Y. H., Zhang, C. Y., Nimmakayalu, M. A., Ward, D. C., and Weissman, S. (2000). Cloning and characterization of two mouse genes with homology to the yeast Sir2 gene. Genomics 69, 355-369. doi: 10.1006/geno. 2000.6360

Yeung, F., Hoberg, J. E., Ramsey, C. S., Keller, M. D., Jones, D. R., and Frye, R. A., et al. (2004). Modulation of NF-kappaB-dependent transcription and cell survival by the SIRT1 deacetylase. EMBO J. 23, 2369-2380. doi: 10.1038/sj.emboj. 7600244

Conflict of Interest Statement: The author declares that the research was conducted in the absence of any commercial or financial relationships that could be construed as a potential conflict of interest.

Received: 31 March 2013; paper pending published: 08 May 2013; accepted: 01 July 2013; published online: 23 July 2013.

Citation: Duan W (2013) Sirtuins: from metabolic regulation to brain aging. Front. Aging Neurosci. 5:36. doi: 10.3389/fnagi.2013.00036

Copyright (c) 2013 Duan. This is an openaccess article distributed under the terms of the Creative Commons Attribution License, which permits use, distribution and reproduction in other forums, provided the original authors and source are credited and subject to any copyright notices concerning any third-party graphics etc. 